\title{
Sex Differences in Reconstructed Resting-State Functional Brain Networks for Children
}

\author{
Xianglai Yang, Han Zhang* \\ State Grid of China Technology College, Jinan, China \\ Email: *sdzh0315@163.com
}

How to cite this paper: Yang, X.L. and Zhang, H. (2020) Sex Differences in Reconstructed Resting-State Functional Brain Networks for Children. Journal of Biosciences and Medicines, 8, 166-177.

https://doi.org/10.4236/jbm.2020.812016

Received: November 30, 2020

Accepted: December 25, 2020

Published: December 28, 2020

Copyright $\odot 2020$ by author(s) and Scientific Research Publishing Inc. This work is licensed under the Creative Commons Attribution International License (CC BY 4.0).

http://creativecommons.org/licenses/by/4.0/

(c) (i) Open Access

\begin{abstract}
Neuroscience studies have demonstrated that functional differences in human brains between males and females might result in their cognitive and psychological distinctions. To investigate sex differences in resting-state functional networks for children, the functional brain networks of two groups including boys and girls were reconstructed by functional connectivity with significant between-group differences respectively based on two brain atlases, and then the reconstructed functional networks were compared from the viewpoint of small-world properties. The functional brain networks of the two groups both displayed topological properties of the small-world network based on different brain atlases but exhibited some sex differences in certain measures. Specifically, for the automated anatomical labeling atlas, compared with girls, boys showed stronger small-world properties and higher ability of local information processing in brain networks; for the Harvard Oxford Atlas, the shortest path length of boys increased, indicating poorer performance in both global information transmission and resistance to the random attack.
\end{abstract}

\section{Keywords}

Sex Difference, Functional Connectivity, Brain Network, fMRI

\section{Introduction}

In recent decades, researchers have paid more attention to sex differences based on functional magnetic resonance imaging (fMRI) for its impressive reflection of spontaneous and intrinsic neural activity, which is related to a variety of cognitive processes [1]. Previous studies based on neuroimaging technologies have demonstrated that the human brain is a complex network consisting of numerous regions with inter-connectivity and sex differences [2]. As a frequently-used technique in neuroscience, resting-state fMRI (rs-fMRI) has the advantage of 
revealing the activity in various brain regions noninvasively by measuring changes of blood oxygen level dependent (BOLD) flow [3]. With the rapid development of neurological research, functional connectivity (FC) has been extensively used in rs-fMRI to reveal the neural mechanism [4] [5]. The functional differences in human brains between males and females might result in their cognitive and psychological distinctions to some extent [6] [7] [8]. The seed-based FC study and independent component analysis (ICA) detected stronger connectivity in the posterior cingulate cortex (PCC), medial prefrontal cortex (mPFC), and inferior parietal lobule (IPL) but lower in the dorsal anterior cingulated cortex (dACC), superior temporal gyrus (STG), insula, and occipital lobe in females [4]. Moreover, stronger FC was observed between the right amygdala and the sensorimotor cortex in females, and lower FC was reported between the left amygdala and the subgenual cortex in males [5].

However, many of the rs-fMRI findings focused more on adults but little on children. Previous electroencephalogram research demonstrated that boys showed higher absolute energy and relative energy in the frequency band of Alpha 1-12 than girls [9], and boys were not developing as quickly as girls of the same age [10]. Nevertheless, there are few studies about sex differences of brain networks based on rs-fMRI for children. More importantly, sex differences change continuously with age [9], and the incidence of certain psychological and emotional disorders differs dramatically in boys and girls [10] [11]. Hence, sex divisions in functional brain networks for children need to be explored further.

For the purpose of investigating complex functional networks of the human brain, small-world properties of the graph theory have been introduced to measure the topological attributes of brain networks [12]. The constructed resting-state functional brain network presented high global efficiency and short characteristic path length, which showed topological properties of the small-world network [13]. Besides, the most significant small-world properties of functional networks were found in the low frequency band of $0.03-0.06 \mathrm{~Hz}$ [14]. What is more, the small-world property of clustering coefficient within the right hemispheric network formed by inter-regional temporal correlations was higher in males than in females, proving the existence of sex-hemisphere interaction [15].

In this current study, the functional brain networks of two children groups (boys and girls) were reconstructed by FC with significant between-group differences respectively based on the automated anatomical labeling (AAL) atlas and the Harvard Oxford Atlas (HOA), and then were analyzed from the viewpoint of small-world properties of the graph theory, in order to investigate sex differences in resting-state functional networks for children.

\section{Materials and Methods}

\subsection{Data Acquisition and Preprocessing}

The rs-fMRI data was acquired from the 1000 Functional Connectomes Project (http://www.nitrc.org/projects/fcon_1000/), with 16 subjects (both 8 boys and 8 
girls with the average age of 12) included. The resting-state functional images were obtained from a 3.0-Tesla scanner (Siemens TRIO TIM, Munich, Germany) with the following parameters: repetition time $(\mathrm{TR})=3000 \mathrm{~ms}$, echo time $(\mathrm{TE})=30 \mathrm{~ms}$, flip angle $(\mathrm{FA})=90^{\circ}$, matrix size $=64 \times 64$, voxel size $=3.75 \times$ $3.75 \times 3.75 \mathrm{~mm}^{3}$ and 120 time points. During the process of data acquisition, all subjects were required to close eyes without head motion or thinking and required to keep awake. Detailed demographic information of subjects was shown in Table 1.

The rs-fMRI data of 16 subjects were preprocessed by Statistical Parametric Mapping (SPM8) toolbox running on MATLAB 2015b. Detailed procedures consisted of format conversion, data elimination, realignment, slice timing, co-registration \& normalization, and smoothing with the $8 \times 8 \times 8 \mathrm{~mm}^{3}$ full-width-at-half-maximum Gaussian kernel.

\subsection{Functional Networks Reconstruction}

The time series of the two groups (boys and girls) were extracted using DPABI toolbox (http://rfmri.org/dpabi) running on MATLAB 2015b based on 90 regions of interests (ROIs) of the AAL atlas and 112 ROIs of the HOA respectively. Pearson correlation coefficients were calculated as FC between the predefined ROIs, and then FC matrixes of each group were generated by Fisher r-to-z transformation. The ROIs with significant between-group differences of FC in both AAL and HOA were selected to construct weighted undirected functional networks separately. The sparsity thresholds $(0.2-0.3)$ were determined according to the principle that the small-worldness parameter should be more than $1.1[16]$.

\subsection{Small-World Properties}

The main parameters of small-word properties in this work included the clustering coefficient $(C P)$, characteristic path length $(L p), \gamma=C p_{\text {real }} / C p_{\text {rand }}>1$, $\lambda=C p_{\text {real }} / C p_{\text {rand }} \approx 1$ [17], $\sigma=\gamma / \lambda>1$ [17], where $C p_{\text {real }}$ and $C p_{\text {rand }}$, $L p_{\text {real }}$ and $L p_{\text {rand }}$ represent $C p, L p$ of the network to be investigated and the random network respectively. The parameter $\sigma$ is directly proportional to the small-worldness; $C P$ represents the degree that nodes in a network incline to cluster together; $L p$ is defined as the number of edges in the shortest path between nodes [13] [17]. In addition, $C p$ and local efficiency $\left(E_{i_{-} \text {local }}\right), L p$ and

Table 1. Demographic characteristics of subjects.

\begin{tabular}{ccc}
\hline \multirow{2}{*}{ Variables } & \multicolumn{2}{c}{ Subjects } \\
\cline { 2 - 3 } & Boys & Girls \\
\hline Age (year) (Mean $\pm \mathrm{SD})$ & $12 \pm 1.32$ & $12 \pm 1.32$ \\
Handedness (Right/Left) & $8 / 0$ & $8 / 0$
\end{tabular}

SD. standard deviation. 
gobal efficiency ( $E_{\text {global }}$ ) were used to measure the performance of the local and global information transmission in the brain separately. $E_{\text {local }}$ is defined as the mean local efficiency of a graph while $E_{\text {global }}$ is calculated as the reciprocal value of the harmonic mean of the minimum path length between every two nodes [18]:

$$
\begin{aligned}
E_{\text {local }} & =(1 / G) \sum_{i \in G} E_{i_{-} \text {local }}, \\
E_{\text {global }} & =\frac{1}{N(N-1)} \sum_{i \neq j \in G} \frac{1}{L_{i, j}},
\end{aligned}
$$

where $E_{i_{-} \text {local }}$ is the local efficiency of the node $i$ in a network $G(N \times N, N=90$ or 112), $L_{i, j}$ is the minimum path length between node $i$ and $j$.

\subsection{Statistical Analysis}

The two-sample two-tailed t-tests were performed on the FC matrixes based on AAL and HOA respectively in order to select the ROIs with significant between-group differences of FC. Then same regions from the selected ROIs based on two-scale brain atlases were chosen again to reconstruct functional networks. The two-sample t-tests were performed on the small-world parameters of the two reconstructed networks based on the reselected ROIs under AAL and HOA separately to uncover sex differences in small-world properties of reconstructed functional brain networks for children.

\section{Results}

\subsection{Identification of ROIs with Significant Between-Group Differences of FC}

The FC matrixes were generated from Pearson correlation coefficients by Fisher r-to-z transformation of the two groups (boys and girls) based on 90 ROIs of AAL and 112 ROIs of HOA were shown in Figure 1 and Figure 2 respectively.

After two-sample t-tests on the FC matrixes based on AAL and HOA separately, the 24 ROIs of AAL in Figure 3(a) and 11 ROIs of HOA in Figure 3(b) showed significant differences $(\mathrm{p}<0.01)$ between boys and girls. Among the ROIs with significant between-group differences $(p<0.01)$ based on AAL and $\mathrm{HOA}$, there were shared areas involving the right heschls gyrus, lingual gyrus, temporal pole, inferior temporal gyrus and parahippocampal gyrus, the left thalamus, temporal pole and inferior temporal gyrus in 9 ROIs of AAL and 8 ROIs of HOA, which was listed in Table 2 and visualized in Figure 3(c).

\subsection{Comparison of Small-World Properties of Reconstructed Functional Brain Networks between Boys and Girls}

The five parameters including $C p, L p, \sigma, \lambda, \gamma$ of small-world properties of the reconstructed functional brain networks for boys and girls were calculated based on AAL $(0.2 \leq$ sparsity $\leq 0.3)$ and HOA $(0.3 \leq$ sparsity $\leq 0.4)$ respectively, which shown in Figure $4 \&$ Figure 5. The three parameters $\sigma>1, \lambda \approx 1, \gamma>1$ indicated 


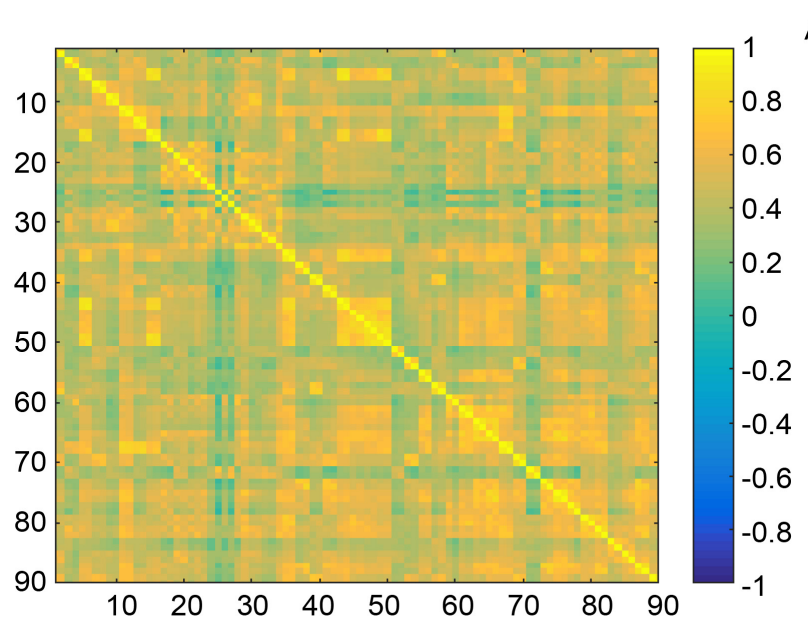

(a) Boys
AAL

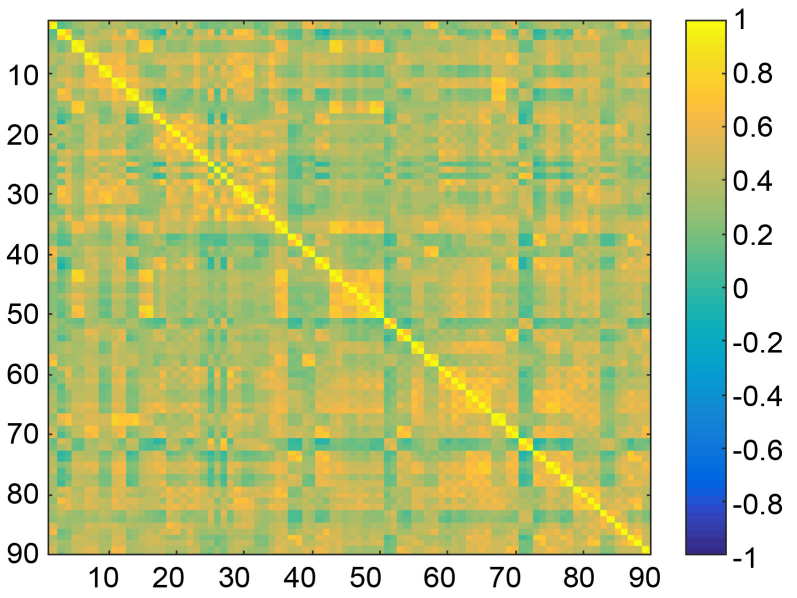

(b) Girls

Figure 1. The FC matrixes based on 90 ROIs of AAL. (a) and (b) showed FC matrixes based on AAL of the boy group and the girl group respectively.

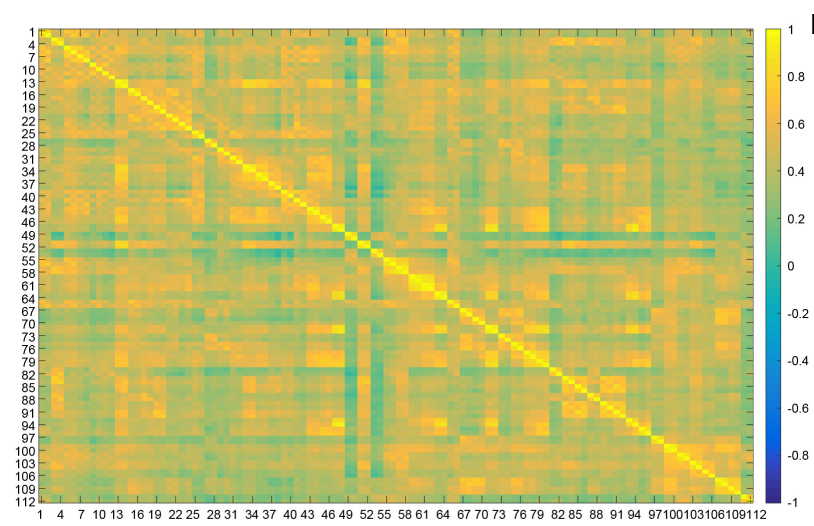

(a) Boys

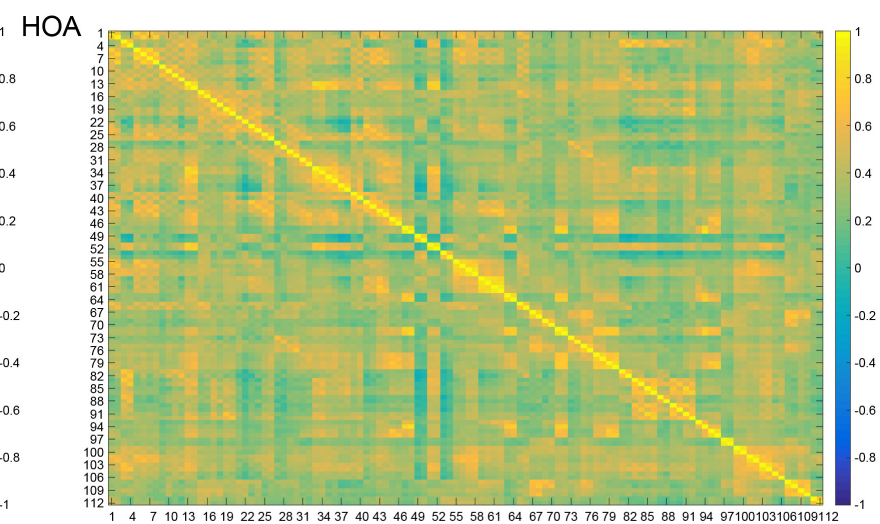

(b) Girls

Figure 2. The FC matrixes based on 112 ROIs of HOA. (a) and (b) showed FC matrixes based on HOA of the boy group and the girl group respectively.

that both of the two groups displayed topological properties of the small-world network based on the two brain atlases.

The two-sample t-tests were performed on the parameters under sparsity of 0.2 - 0.4, the two groups exhibited some sex differences in certain measures based on both of the two brain atlases. For AAL, $C P(0.20 \leq$ sparsity $\leq 0.30)$ and $L p, \sigma, \lambda, \gamma(0.20 \leq$ sparsity $\leq 0.25)$ showed significant between-group differences $(\mathrm{p}<0.05)$. For HOA, $\lambda(0.30 \leq$ sparsity $\leq 0.40)$ showed significant between-group differences $(\mathrm{p}<0.05)$ but $C P, L p$ did not differ statistically between boys and girls. To be specific, for AAL, increased $C p(0.20 \leq$ sparsity $\leq 0.27), \sigma$ (0.20 $\leq$ sparsity $\leq 0.25), \lambda(0.20 \leq$ sparsity $\leq 0.25), \gamma(0.20 \leq$ sparsity $\leq 0.25), L p$ $(0.20 \leq$ sparsity $\leq 0.25)$ were observed in boys; for HOA, enhanced $\lambda(0.30 \leq$ sparsity $\leq 0.40), \sigma(0.30 \leq$ sparsity $\leq 0.35), \gamma(0.30 \leq$ sparsity $\leq 0.35)$ were detected in boys. 
(a)

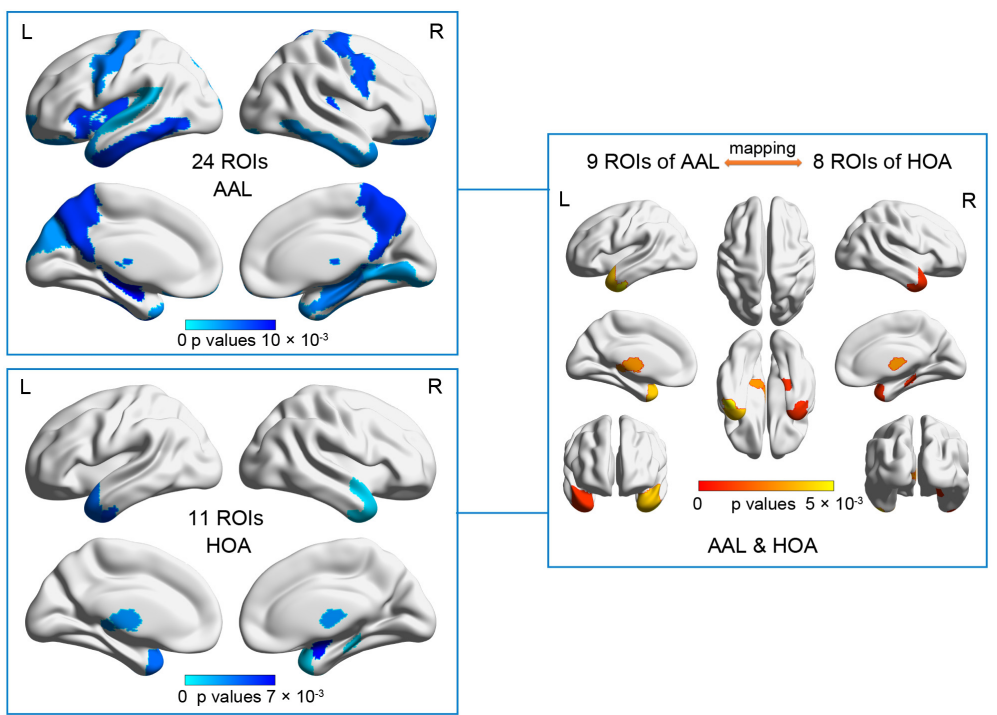

(c)

Figure 3. The ROIs with significant between-group differences of FC. (a) and (b) showed ROIs with significant between-group differences based on AAL and HOA respectively, and (c) shows regions in both 9 ROIs of AAL and 8 ROIs of HOA.

\section{Discussions}

In this study, a total of 9 ROIs of AAL corresponding to 8 ROIs of HOA were detected with significant between-group differences of FC and then were selected to reconstruct functional brain networks based on AAL and HOA for the boy group and girl group. The small-world properties of the brain networks were compared between boys and girls to further explore sex differences in resting-state functional networks for children.

The shared regions in both 9 ROIs of AAL and 8 ROIs of HOA showed significant between-group differences of FC, including the right heschls gyrus, the right lingual gyrus, the right temporal pole, the right inferior temporal gyrus, the right parahippocampal gyrus, the left thalamus, the left temporal pole, and the left inferior temporal gyrus. Previous studies have demonstrated that some of these shared areas are related to sex differences in language, memory, visual-spatial cognitive ability and emotion-focused coping [19] [20] [21]. As a crucial role of the ventral visual pathway, the inferior temporal gyrus mainly participates in face recognition and memory [22]. It has been reported that males show stronger activation in the left infratemporal gyrus than females [23]. The differences in FC of the left inferior temporal gyrus between boys and girls during resting state in this study may be the basis of sex differences in the activity of the temporal lobe during certain cognitive tasks. The parahippocampal gyrus, relating to memory and emotional processing in the limbic system, can accept the input of perceptual information and then transmit it to the hippocampus [24]. It has been found that the left parahippocampal gyrus of males is more activated during a language fluency test than that of females [25]. The differences in resting-state FC of the parahippocampal gyrus between boys and girls in this work revealed that there were sex differences in the neurological basis of the 

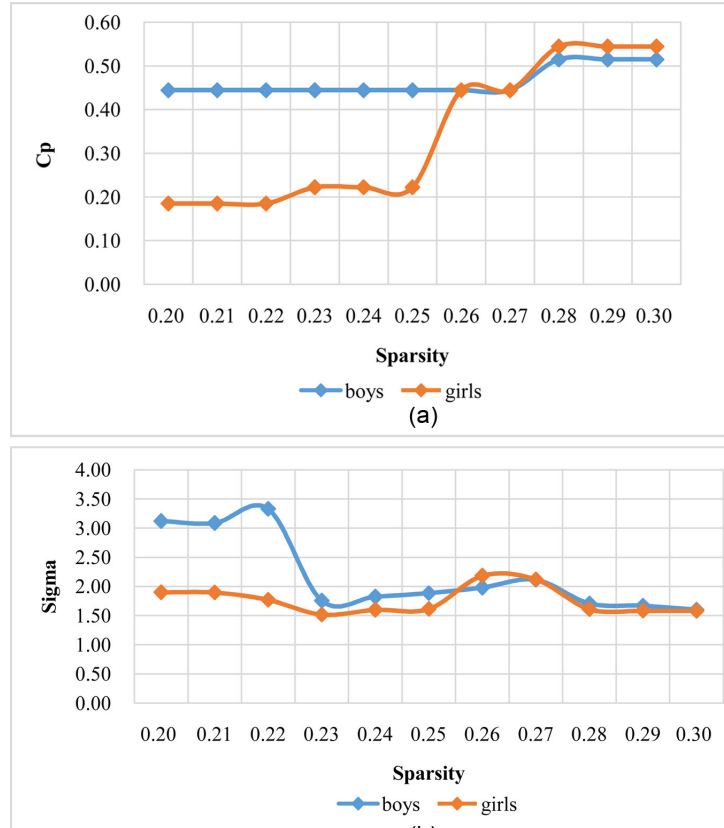

(b)

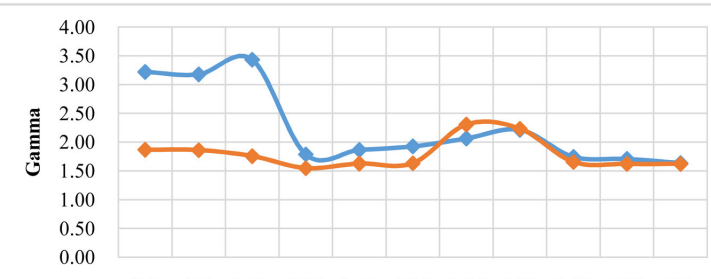

$\begin{array}{lllllllllll}0.2 & 0.21 & 0.22 & 0.23 & 0.24 & 0.25 & 0.26 & 0.27 & 0.28 & 0.29 & 0.3\end{array}$ Sparsity

$\multimap$ boys $\leadsto$ girls

(c)

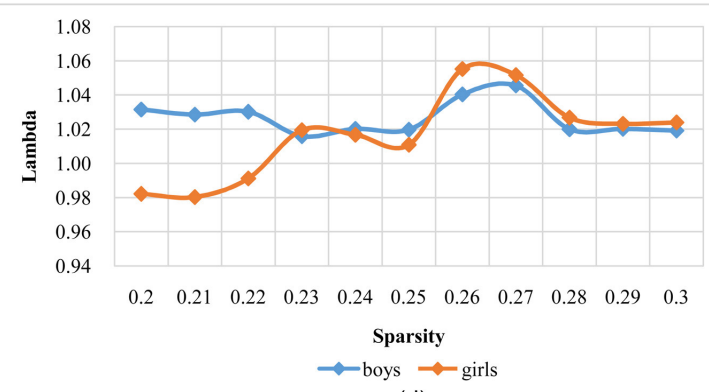

(d)

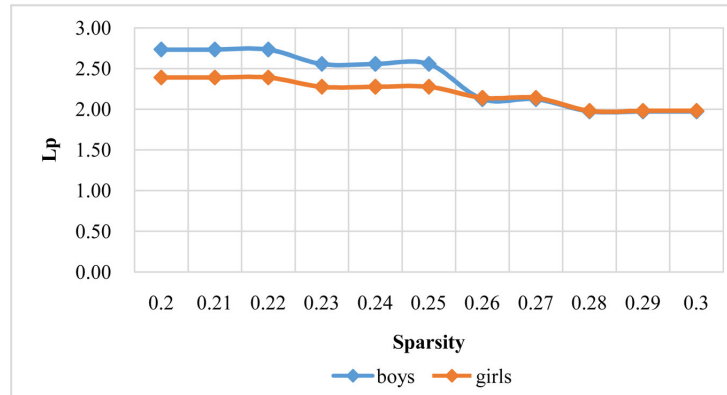

(e)

Figure 4. Small-world properties of the two groups based on AAL. (a)-(e) showed the five parameters of $C p, L p, \sigma$, $\lambda$, and $\gamma$ respectively based on AAL. 

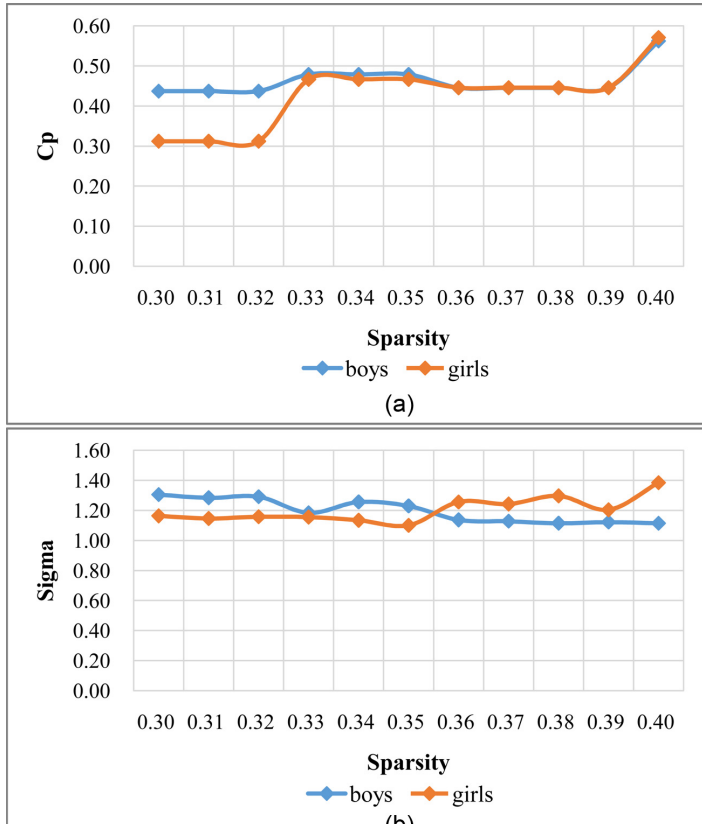

(b)

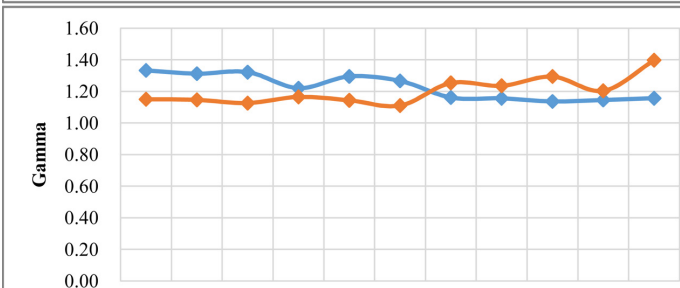

$\begin{array}{lllllllllll}0.30 & 0.31 & 0.32 & 0.33 & 0.34 & 0.35 & 0.36 & 0.37 & 0.38 & 0.39 & 0.40\end{array}$ Sparsity

$\multimap$ boys $\leadsto$ girls

(c)

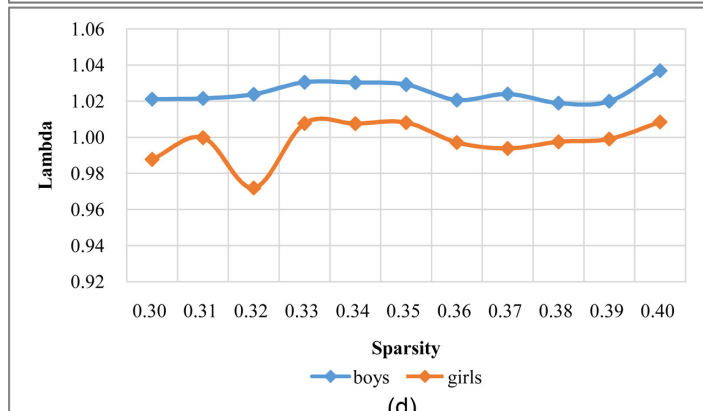

(d)

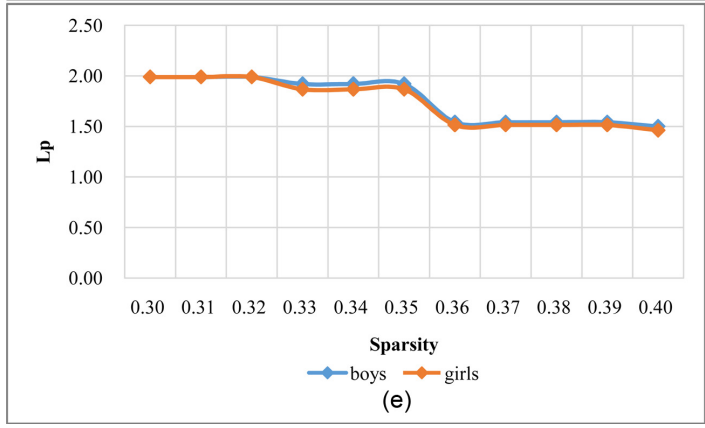

Figure 5. Small-world properties of the two groups based on HOA. (a)-(e) showed the five parameters of $C p, L p, \sigma, \lambda$, and $\gamma$ respectively based on HOA. 
Table 2. The ROIs with significant between-group differences of FC based on both AAL and HOA.

\begin{tabular}{clcc}
\hline \multicolumn{1}{c}{ Atlas } & \multicolumn{1}{c}{ Region } & p value & Hemisphere \\
\hline \multirow{2}{*}{ AAL } & Temporal pole: superior temporal gyrus & $2.46 \mathrm{E}^{-05}$ & $\mathrm{R}$ \\
& Lingual gyrus & 0.0031 & $\mathrm{R}$ \\
& Temporal pole: middle temporal gyrus & 0.0035 & $\mathrm{R}$ \\
& Inferior temporal gyrus & 0.0036 & $\mathrm{R}$ \\
& Temporal pole: middle temporal gyrus & 0.0041 & $\mathrm{~L}$ \\
& Parahippocampal gyrus & 0.0049 & $\mathrm{R}$ \\
& Thalamus & 0.0072 & $\mathrm{~L}$ \\
& Heschl gyrus & 0.0078 & $\mathrm{R}$ \\
& Inferior temporal gyrus & 0.0084 & $\mathrm{~L}$ \\
\hline Heschls gyrus (includes H1, H2) & $9.12 \mathrm{E}^{-05}$ & $\mathrm{R}$ \\
& Lingual gyrus & 0.0003 & $\mathrm{R}$ \\
& Temporal pole & 0.0006 & $\mathrm{R}$ \\
Inferior temporal gyrus, anterior division & 0.0007 & $\mathrm{R}$ \\
Parahippocampal gyrus, posterior division & 0.0009 & $\mathrm{R}$ \\
Thalamus & 0.0025 & $\mathrm{~L}$ \\
Temporal pole & 0.0035 & $\mathrm{~L}$ \\
Inferior temporal gyrus, anterior division & 0.0054 & $\mathrm{~L}$ \\
\hline
\end{tabular}

ability to perceive things and transmit information. The thalamus is one of the key regions in the pathway of diverse perceptual information to the cerebral cortex [26]. Studies have indicated that thalamo-cortical FC of males is significantly stronger than that of females [27] [28], which is basically consistent with the results of this research. It further proves that there are differences in the activity of the thalamus between boys and girls during the transmission of perceptual information.

For small-world properties of the reconstructed functional brain networks, sex differences were detected based on both AAL and HOA. Compared with girls, boys showed stronger small-world properties by increased $\sigma$, higher ability of information integration and transmission by increased $C p$, higher ability of global information processing in brain networks by increased $\lambda$, higher efficiency for local information processing by enhanced $\gamma$, as well as poorer performance in both global information transmission and resistance to the random attack by enhanced $L p$. Briefly, for AAL, boys showed stronger small-world properties and higher ability of local information processing in brain networks; for HOA, girls showed better performance in both global information transmission and resistance to the random attack.

It is universally acknowledged that the human brain performs various functions of constantly monitoring the internal and external environment, situational memory, as well as continuous cognitive interpretation during the resting state [29]. The differences of cognitive and behavioral manifestations in boys 
and girls partly result from the neurological activity within the brain, thus this study may help to further understand sex differences in functional brain networks for children.

\section{Conclusion}

In this work, the calculated FC based on AAL and HOA showed significant between-group differences among 9 ROIs of AAL and 8 ROIs of HOA. The functional brain networks reconstructed by the FC among these ROIs were analyzed from the viewpoint of small-world properties. The boys showed stronger small-world properties and higher ability of local information processing in brain networks while the girls performed better in global information transmission and resistance to the random attack.

\section{Limitation}

This study was based on a small-sample dataset including the fMRI data of 16 children, therefore the universality of the conclusion needs to be proved on a larger-sample dataset in the future work and the specific cognitive meaning behind the results needs further exploration.

\section{Conflicts of Interest}

The authors declare no conflicts of interest regarding the publication of this paper.

\section{References}

[1] Gusnard, D.A. and Raichle, M.E. (2001) Searching for a Baseline: Functional Imaging and the Resting Human Brain. Nature Reviews Neuroscience, 2, 685-694. https://doi.org/10.1038/35094500

[2] Allen, J.S., Damasio, H., Grabowski, T.J., Bruss, J. and Zhang, W. (2003) Sexual Dimorphism and Asymmetries in the Gray-White Composition of the Human Cerebrum. NeuroImage, 18, 880-894. https://doi.org/10.1016/S1053-8119(03)00034-X

[3] Kwong, K.K., Belliveau, J.W., Chesler, D.A., et al. (1992) Dynamic Magnetic Resonance Imaging of Human Brain Activity during Primary Sensory Stimulation. Proceedings of the National Academy of Sciences of the United States of America, 89, 5675-5679. https://doi.org/10.1073/pnas.89.12.5675

[4] Biswal, B.B., Mennes, M., Zuo, X.N., et al. (2010) Toward Discovery Science of Human Brain Function. Proceedings of the National Academy of Sciences of the United States of America, 107, 4734-4739. https://doi.org/10.1073/pnas.0911855107

[5] Kilpatrick, L.A., Zald, D.H., Pardo, J.V. and Cahillab, L.F. (2006) Sex-Related Differences in Amygdala Functional Connectivity during Resting Conditions. NeuroImage, 30, 452-461. https://doi.org/10.1016/j.neuroimage.2005.09.065

[6] Gur, R.C., Turetsky, B.I., Matsui, M., et al. (1999) Sex Differences in Brain Gray and White Matter in Healthy Young Adults: Correlations with Cognitive Performance. Neuroscience, 19, 4065-4072. https://doi.org/10.1523/JNEUROSCI.19-10-04065.1999 
[7] Cragg, L., Kovacevic, N., Mcintosh, A.R., et al. (2011) Maturation of EEG Power Spectra in Early Adolescence: A Longitudinal Study. Development Sciences, 14, 935-943. https://doi.org/10.1111/j.1467-7687.2010.01031.x

[8] Matthis, P., Scheffner, D., Benninger, C., Lipinski, C. and Stolzis, L. (1980) Changes in the Background Activity of the Electroencephalogram According to Age. Electroencephalography and Clinical Neurophysiology, 49, 626-635. https://doi.org/10.1016/0013-4694(80)90403-4

[9] Gong, G., He, Y. and Evans, A.C. (2011) Brain Connectivity: Gender Makes a Difference. The Neuroscientist, 17, 575-591. https://doi.org/10.1177/1073858410386492

[10] Scott, F.J., Baron-Cohen, S., Bolton, P. and Brayne, C. (2002) Brief Report Prevalence of Autism Spectrum Conditions in Children Aged 5-11 Years in Cambridgeshire, UK. Autism, 6, 231-237. https://doi.org/10.1177/1362361302006003002

[11] Corrigan, N.M., Richards, T.L., Treffert, D.A. and Dager, S.R. (2012) Toward a Better Understanding of the Savant Brain. Comprehensive Psychiatry, 53, 706-717. https://doi.org/10.1016/j.comppsych.2011.11.006

[12] Salcador, R., Suckling, J., Coleman, M.R., et al. (2005) Neurophysiological Architecture of Functional Magnetic Resonance Images of Human Brain. Cerebral Cortex, 15, 1332-1342. https://doi.org/10.1093/cercor/bhi016

[13] Watts, D.J. and Strongatz, S.H. (1998) Collective Dynamics of 'Small-World' Networks. Nature, 393, 440-442. https://doi.org/10.1038/30918

[14] Achard, S., Salvador, R., Whitcher, B., Suckling, J. and Bullmore, E. (2006) A Resilient, Low-Frequency, Small-World Human Brain Functional Network with Highly Connected Association Cortical Hubs. Journal of Neuroscience, 26, 63-72. https://doi.org/10.1523/JNEUROSCI.3874-05.2006

[15] Tian, L., Wang, J., Yan, C., Yan, C. and He, Y. (2011) Hemisphere and Gender-Related Differences in Small World Brain Networks: A Resting-State Functional MRI Study. NeuroImage, 54, 191-202. https://doi.org/10.1016/j.neuroimage.2010.07.066

[16] Mecherlli, A., Friston, K.J., Frackowiak, R.S. and Price, C.J. (2005) Structural Covariance in the Human Cortex. Journal of Neuroscience, 25, 8303-8310. https://doi.org/10.1523/JNEUROSCI.0357-05.2005

[17] Humphries, M.D., Gurney, K. and Prescott, T.J. (2006) The Brainstem Reticular Formation is a Small World, Not Scale-Free, Network. Proceedings of the Royal Society B-Biological Sciences, 273, 503-511. https://doi.org/10.1098/rspb.2005.3354

[18] Achard, S. and Bullmore, E. (2007) Efficiency and Cost of Economical Brain Functional Networks. PLoS Computational Biology, 3, e17. https://doi.org/10.1371/journal.pcbi.0030017

[19] Xu, C., Li, C., Wu, H., Wu, Y. and Zhang, X. (2015) Gender Differences in Cerebral Regional Homogeneity of Adult Healthy Volunteers: A Resting-State FMRI Study. Journal of Biomedicine and Biotechnology, 2015, Article ID: 183074. https://doi.org/10.1155/2015/183074

[20] Rizk-Jackson, A.M., Acevedo, S.F., Inman, D., et al. (2006) Effects of Sex on Object Recognition and Spatial Navigation in Humans. Behavioural Brain Research, 173, 181-190. https://doi.org/10.1016/j.bbr.2006.06.029

[21] Saykin, A.J., Johnson, S.C., Flashman, L.A., et al. (1999) Functional Differentiation of Medial Temporal and Frontal Regions Involved in Processing Novel and Familiar Words: An fMRI Study. Brain, 122, 1963-1971. 
https://doi.org/10.1093/brain/122.10.1963

[22] Haxby, J.V., Hoffman, E.A. and Gobbini, M.I. (2000) The Distributed Human Neural System for Face Perception. Trends in Cognitive Sciences, 4, 223-233. https://doi.org/10.1016/S1364-6613(00)01482-0

[23] Grabowski, T.J., Damasio, H., Eichhorn, G.R. and Tranel, D. (2003) Effects of Gender on Blood Flow Correlates of Naming Concrete Entities. NeuroImage, 20, 940-954. https://doi.org/10.1016/S1053-8119(03)00284-2

[24] Burwell, R.D. (2000) The Parahippocampal Region: Corticocortical Connectivity. Annals of the New York Academy of Sciences, 911, 25-42. https://doi.org/10.1111/j.1749-6632.2000.tb06717.x

[25] Chambers, C.D., Payne, J.M., Stokes, M.G. and Mattingley, J.B. (2004) Fast and Slow Parietal Pathways Mediate Spatial Attention. Nature Neuroscience, 7, 217-218. https://doi.org/10.1038/nn1203

[26] Sherman, S.M. and Guillery, R.W. (2002) The Role of the Thalamus in the Flow of Information to the Cortex. Philosophical Transactions of the Royal Society B-Biological Sciences, 357, 1695-1708. https://doi.org/10.1098/rstb.2002.1161

[27] Cowan, R.L., Rainey, M., Levin, J.M., et al. (2000) Sex Differences in Response to Red and Blue Light in Human Primary Visual Cortex: A Bold fMRI Study. Psychiatry Research, 100, 129-138. https://doi.org/10.1016/S0925-4927(00)00074-3

[28] Bell, E.C., Willson, M.C., Wilman, A.H., Dave, S. and Silverstone, P.H. (2006) Males and Females Differ in Brain Activation during Cognitive Tasks. NeuroImage, 30, 529-538. https://doi.org/10.1016/j.neuroimage.2005.09.049

[29] Raichle, M.E., MacLeod, A.M., Snyder, A.Z., et al. (2001) A Default Mode of Brain Function. Proceedings of the National Academy of Sciences of the United States of America, 98, 676-682. https://doi.org/10.1073/pnas.98.2.676 